\title{
Significant improvement of GAGG:Ce based scintillation detector performance with temperature decrease
}

\author{
M.Korjik ${ }^{1}$, V.Alenkov ${ }^{3}$, A.Borisevich ${ }^{1}$, O.Buzanov ${ }^{3}$, V.Dormenev ${ }^{4}$, G.Dosovitskiy ${ }^{5}$, \\ A.Dosovitskiy ${ }^{6}$, A.Fedorov ${ }^{2}$, D.Kozlov ${ }^{1}$, V.Mechinsky ${ }^{1}$, R.W.Novotny ${ }^{4}$, G.Tamulaitis ${ }^{7}$, \\ V.Vasiliev $^{3}$, H.-G.Zaunick ${ }^{4}$, A. A. Vaitkevičiuss ${ }^{7}$ \\ 1- Research Institute for Nuclear Problems, Minsk, Belarus \\ 2-Radiation Instruments and New Components, Minsk, Belarus \\ 3-Fomos Crystals, Moscow, Russia \\ 4-Justus Liebig University, Giessen, Germany \\ 5-Institute of Chemical Reagents and High Purity Chemical Substances, IREA, Russia \\ 6-NeoChem, Moscow, Russia \\ 7-Vilnius University, Vilnius, Lithuania
}

Abstract - This report presents results on the significant improvement of GAGG:Ce based scintillation detector performance with temperature decrease. When temperature of a PMT based detector is lowered to $-45^{\circ} \mathrm{C}$, its amplitude response at registration of $\gamma$-quanta is improved by $30 \%$; FHHM was found to be better up to factor of 0.85 , whereas scintillation kinetics become even faster in crystals co-doped with magnesium and magnesium and titanium. All this opens an opportunity for a wide application of GAGG scintillation detectors, particularly in a combination with SiPM photo-sensors, which signal-to-noise ratio would also improve with temperature decrease.

Index Terms - Inorganic scintillation material, GAGG scintillator, $\gamma$-quanta, photo-sensor

Corresponding author: Mikhail Korjik (Mikhail.Korjik@cern.ch), 220030, Belarus, Minsk, Bobruiskaya str. 11. 


\section{Introduction}

Due to a high light yield of up to 50000 phot/MeV, a short luminescence decay time $(<100 \mathrm{~ns})$ [1], and good matching of the emission band with the sensitivity spectrum of conventional SiPMs, Ce doped $\mathrm{Gd}_{3} \mathrm{Al}_{2} \mathrm{Ga}_{3} \mathrm{O}_{12}$ crystal (GAGG) is promising scintillation material for medical imaging and might compete with Ce-doped $\mathrm{Lu}_{2} \mathrm{SiO}_{5}$ crystal for PET application. Moreover, GAGG:Ce, co-doped with $\mathrm{Mg}$, shows spectacular time resolution at different excitation [2,3]. GAGG is dense high light yield scntillator, hence it can be applied for a high resolution $\gamma$-radiation spectrometry similar to recently developed halide scintillators [4]. However, wide application of the material in detectors is limited. The material exhibits strong phosphorescence, both under photo-excitation and excitation by ionizing radiation. It is demonstrated that the phosphorescence might be diminished by co-doping of GGAG:Ce crystals with Mg [5]. However, contrary to many other scintillators [6-8] the co-doping of the GAGG material with the second group di-valent ions from the set of $\mathrm{Mg}, \mathrm{Ca}, \mathrm{Sr}$ results in a lower scintillation light yield.

Recently, we showed that light yield deterioration of the crystal at codoping with $\mathrm{Mg}$ is accompanied with a strong acceleration rate of the free carriers non-radiating recombination [9]. This effect becomes competing to a radiating recombination of free carriers via $\mathrm{Ce}^{3+}$ ions and, thus, results in decrease of the scintillator light yield. Non-radiative recombination occurs when hole, due to a migration, appears in the vicinity of the $\mathrm{Mg}^{2+}$ created defect. Moreover, some energy barrier in the vicinity of the recombination center has to be overcome by hole for a recombination. Both, migration rate and an ability to overcome barrier are dependent on the temperature. Thus, possible solution to recuperate light yield loss is a slowing down of the holes mobility. Due to a strong temperature $(\mathrm{T})$ dependence of the both effects $\sim \exp (-\mathrm{E} / \mathrm{kT})$, where $\mathrm{E}$ is the constant, dedicated to migration rate or transmission through the barrier, which are defined by the nature of the compound, k-Bolzman constant, this can be achieved by cooling of the crystal or the whole detecting unit.

Cooling of the scintillation material to gain light yield works pretty good in self-activated scintillation materials which structural units, oxy-anionic complexes, possess highly temperature quenched luminescence. There are a reasonable amount of self-activated materials which includes widely used $\mathrm{PbWO}_{4}$ and $\mathrm{Bi}_{4} \mathrm{Ge}_{3} \mathrm{O}_{12}$ [4]. Note, last modification of $\mathrm{PbWO}_{4}, \mathrm{PWO}$-II, 
which is the crystal, doped with La, and Y at the total amount of less than 100ppm. Doping ions create shallow electron capturing centers in the crystal matrix and prevents free carriers capture by deep traps, significantly improving tolerance of the PWO-II to ionizing radiation. Cooling of the crystal to the $-25^{\circ} \mathrm{C}$ allows to triplicate its light yield at simultaneous keeping the scintillation kinetics fast enough [10].

Contrary to self-activated material, the luminescence of Ce-doped scintillation material is caused by inter-configuration d-f luminescence, having a high quantum yield and low temperature quenching effect in the vicinity of room temperature. Thus, only a minor gain of the light yield of the Ce activated scintillation material is expected with decrease of the crystal temperature. Moreover, some of oxide scintillators doped with $\mathrm{Ce}$, particularity perovskites of $\mathrm{YAlO}_{3}-\mathrm{LuAlO}_{3}$ family, demonstrate $10-20 \%$ decrease of the light yield when temperature is lowered from room to $-20^{\circ} \mathrm{C}$. Here we report an incredible improvement of GAGG:Ce,Mg and GAGG:Ce, $\mathrm{Mg}$, Ti scintillation detectors performance with temperature decrease.

\section{Samples}

All the garnet samples under study were cut from single crystal boules, grown by the Czochralski method from iridium crucibles by Fomos Crystals from the melt close to $\mathrm{Gd}_{3} \mathrm{Al}_{2} \mathrm{Ga}_{3} \mathrm{O}_{12}$ stoichiometric composition. To compensate gallium leakage from the melt during the crystal growth, samples were grown with excessive $\mathrm{Ga}_{2} \mathrm{O}_{3}$ added into the melt in the crucible. The reference sample (S1) was doped with Ce (0.5 at.\%) whereas two other studied crystals, S2 and S3, were co-doped by $\mathrm{Mg}(0.1$ at.\%) and $\mathrm{Mg}$ (0.05 at.\%) and $\mathrm{Ti}(0.01 \mathrm{at} . \%)$ correspondingly. Pulse height spectra of ${ }^{137} \mathrm{Cs}$ source with $662 \mathrm{keV}$ energy of gamma-quanta have been measured with bialkali green-extended PMT Hamamatsu R329 in a thermostat within temperature range from $+20^{\circ} \mathrm{C}$ to $-45^{\circ} \mathrm{C}$, with temperature stabilization accuracy $0.1^{\circ} \mathrm{C}$. Light was measured with bialkali PMT Hamamatsu R2059 in different time gates of charge sensitive gated ADC. Stability of PMT - photocathode sensitivity and PMT gain in the temperature range was controlled by a reference green LED light pulse source. Sample size was $15 \times 18 \times 7 \mathrm{~mm}$. We used Basylon ${ }^{\circledR}$ optical grease and Teflon ${ }^{\circledR}$ light reflectors.

\section{Experimental results and discussion}

Change of the photo-peak position (i.e. light yield change), and change of energy 
104 resolution with temperature were measured in different time gates. Figure 1 represents pulse 105 height spectrum of ${ }^{137} \mathrm{Cs}$ gamma-source measured with S3 sample at four temperatures: $+20^{\circ} \mathrm{C}$, $1060^{\circ} \mathrm{C},-20^{\circ} \mathrm{C},-45^{\circ} \mathrm{C}$. Cooling of the detecting unit results in improvement both, peak position and 107 energy resolution as well. Figure 2 shows change of the normalized to room temperature and 108 measured in 1000 ns gate FWHM of $662 \mathrm{keV}$ photo-peak with temperature. The most 109 prominent improvement has been achieved with the sample co-doped with $\mathrm{Mg}$, whereas Ce 110 doped and Ce doped and co-doped with $\mathrm{Mg}$ and $\mathrm{Ti}$ showed similar behavior.

111

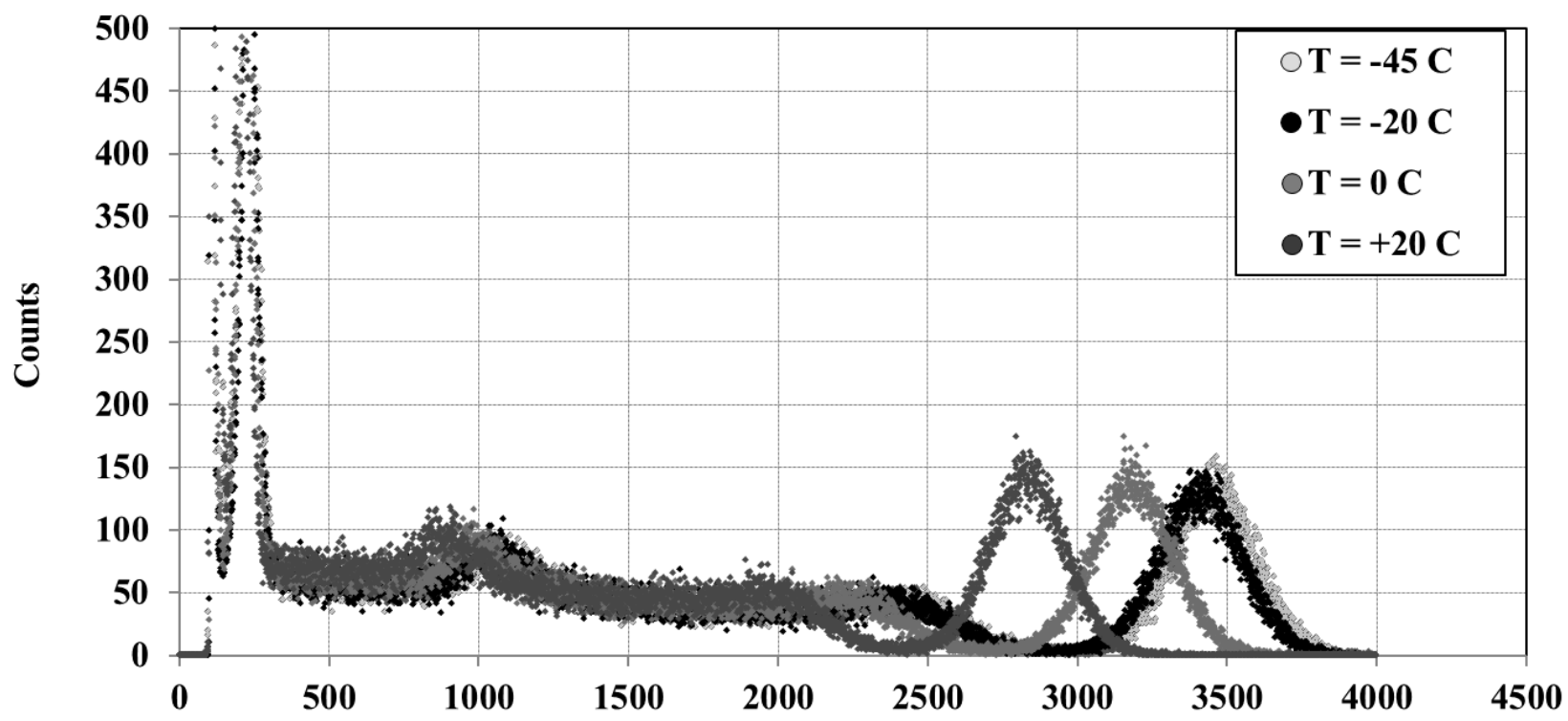

112

113

114

Channel

Fig.1. Pulse height spectrum of ${ }^{137} \mathrm{Cs}$ gamma-source measured with S3 sample at four temperatures: $+20^{\circ} \mathrm{C}, 0^{\circ} \mathrm{C},-20^{\circ} \mathrm{C},-45^{\circ} \mathrm{C}$. 


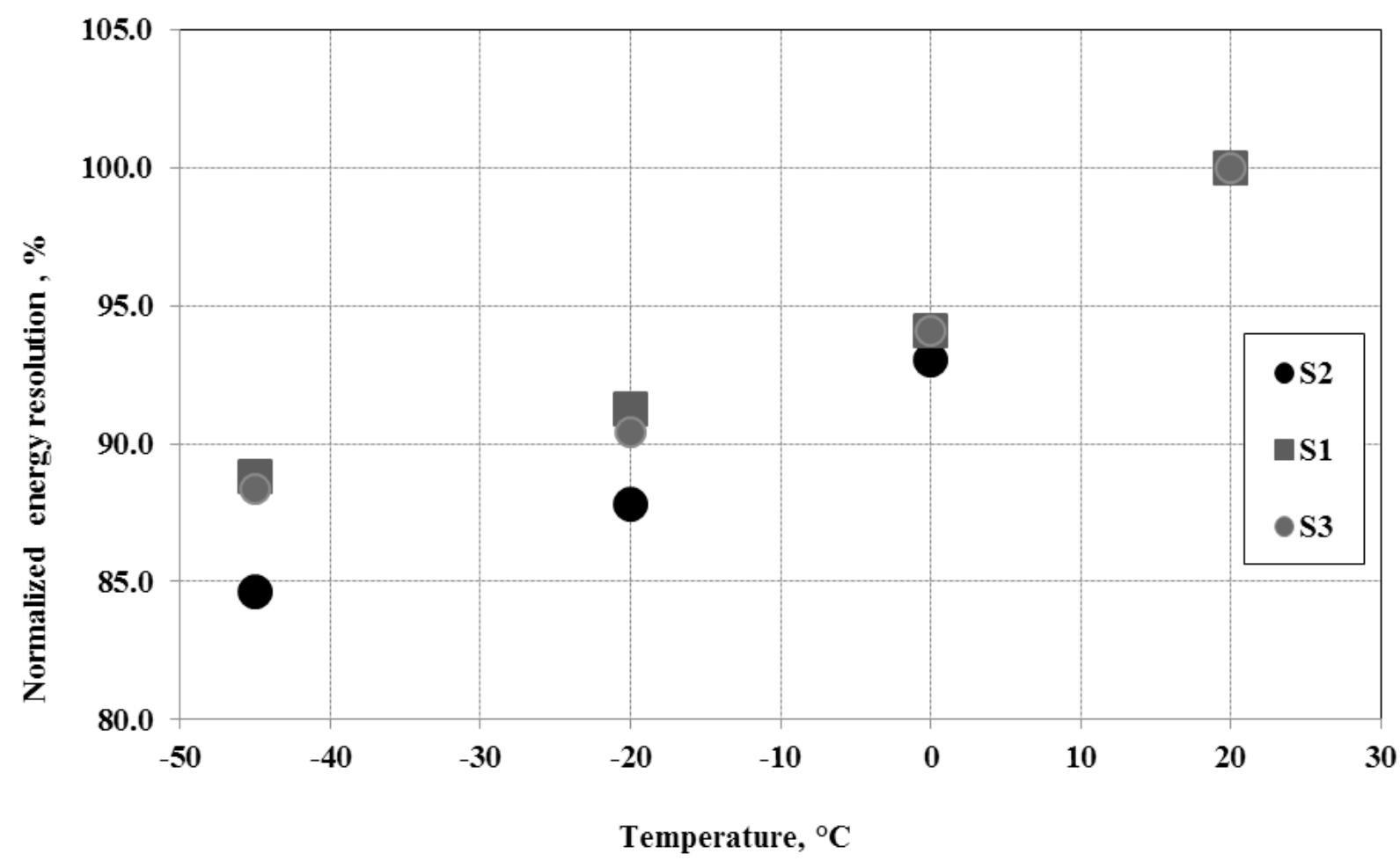

Fig.2. Temperature change of energy resolution FWHM at $662 \mathrm{keV}$ normalized to room temperature. Measurements have been performed with $1000 \mathrm{~ns}$ time gate.

Figures 3-5 show light yield of the samples in various time gates, measured at different temperatures. It worth to note that largest improvement of the LY at cooling is observed in the crystals, co-doped with $\mathrm{Mg}$ and $\mathrm{Mg}$, Ti. Figures 6-8 show relative change of the light yield, normalized to $20^{\circ} \mathrm{C}$, versus time gate at different temperatures. 


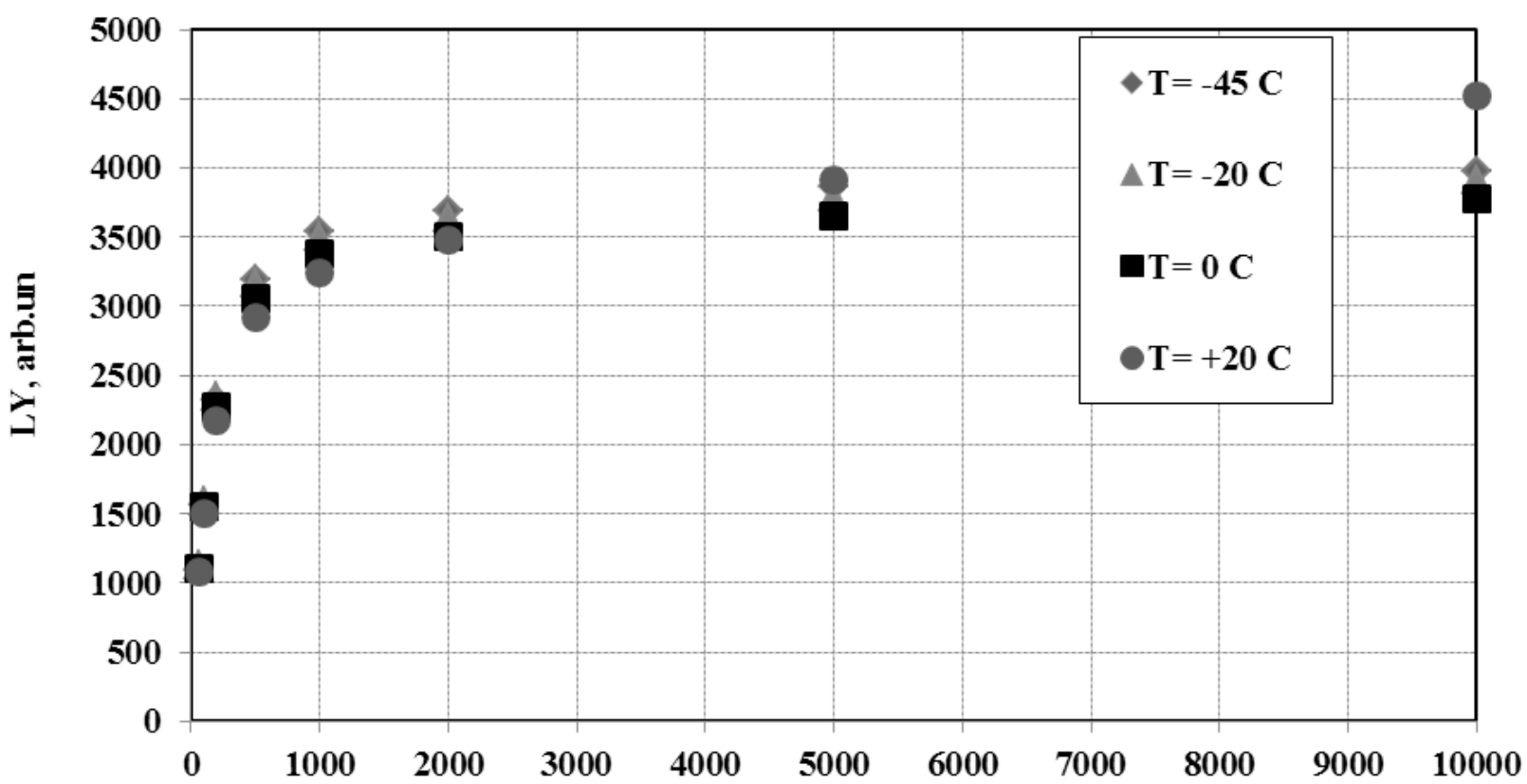

Time gate, ns

Fig.3. Light yield of GAGG:Ce (S1) sample measured in different time gates in temperature range.

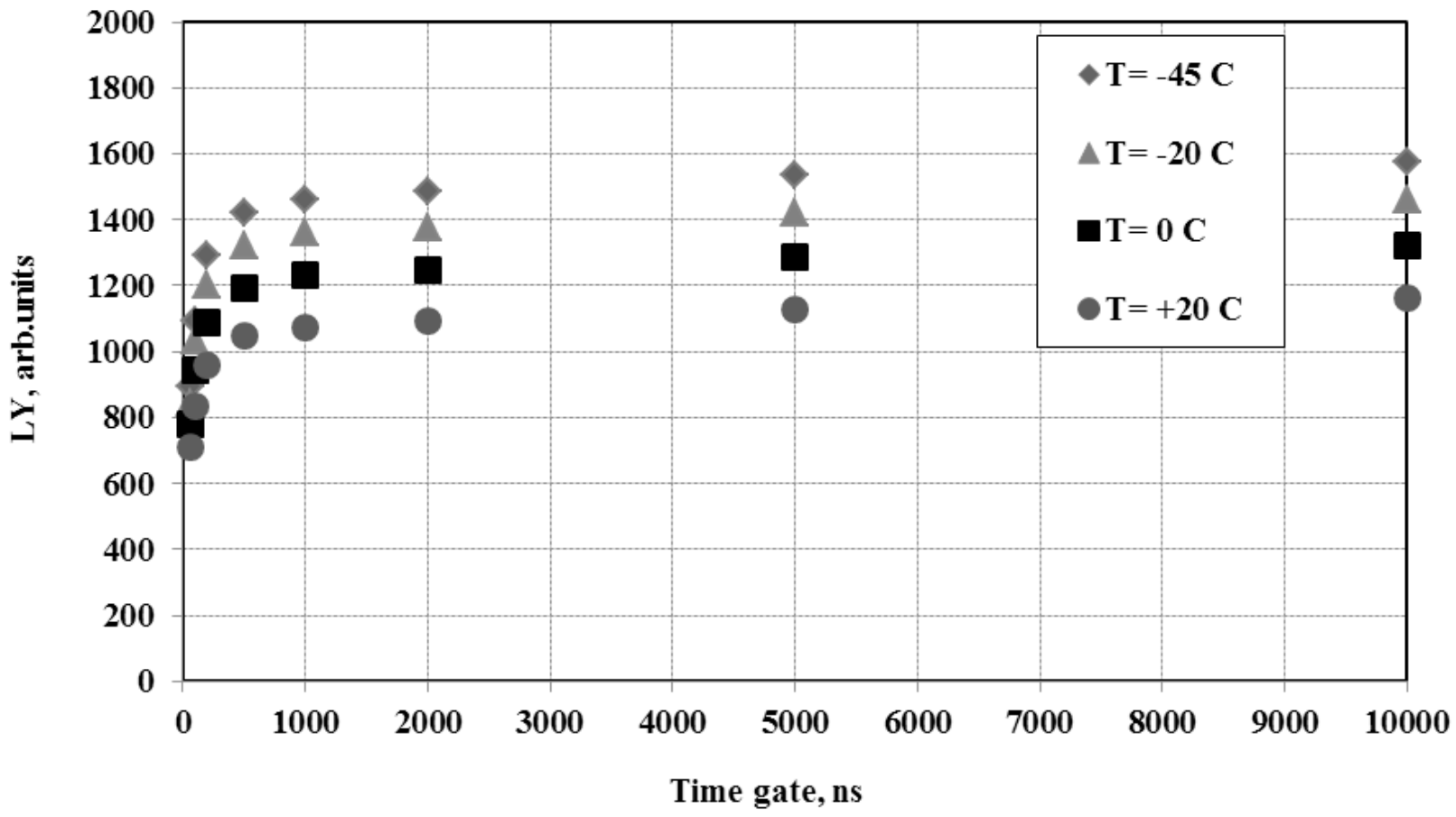

Fig.4. Light yield of GAGG:Ce, $\mathrm{Mg}$ (S2) sample measured in different time gates in 
temperature range.

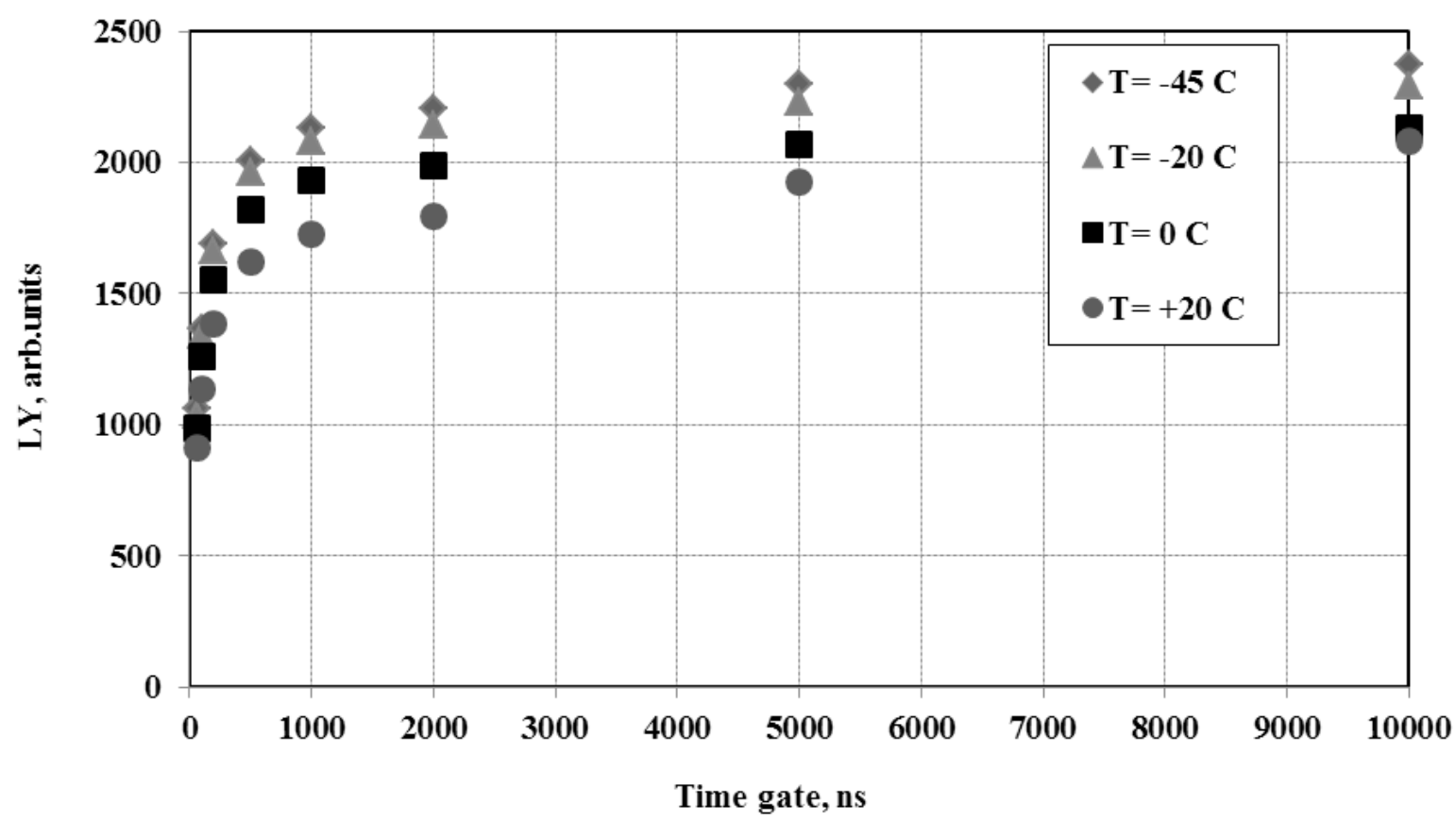

129

130

131

Fig.5. Light yield of GAGG:Ce, Mg, Ti (S3) sample measured in different time gates in temperature range.

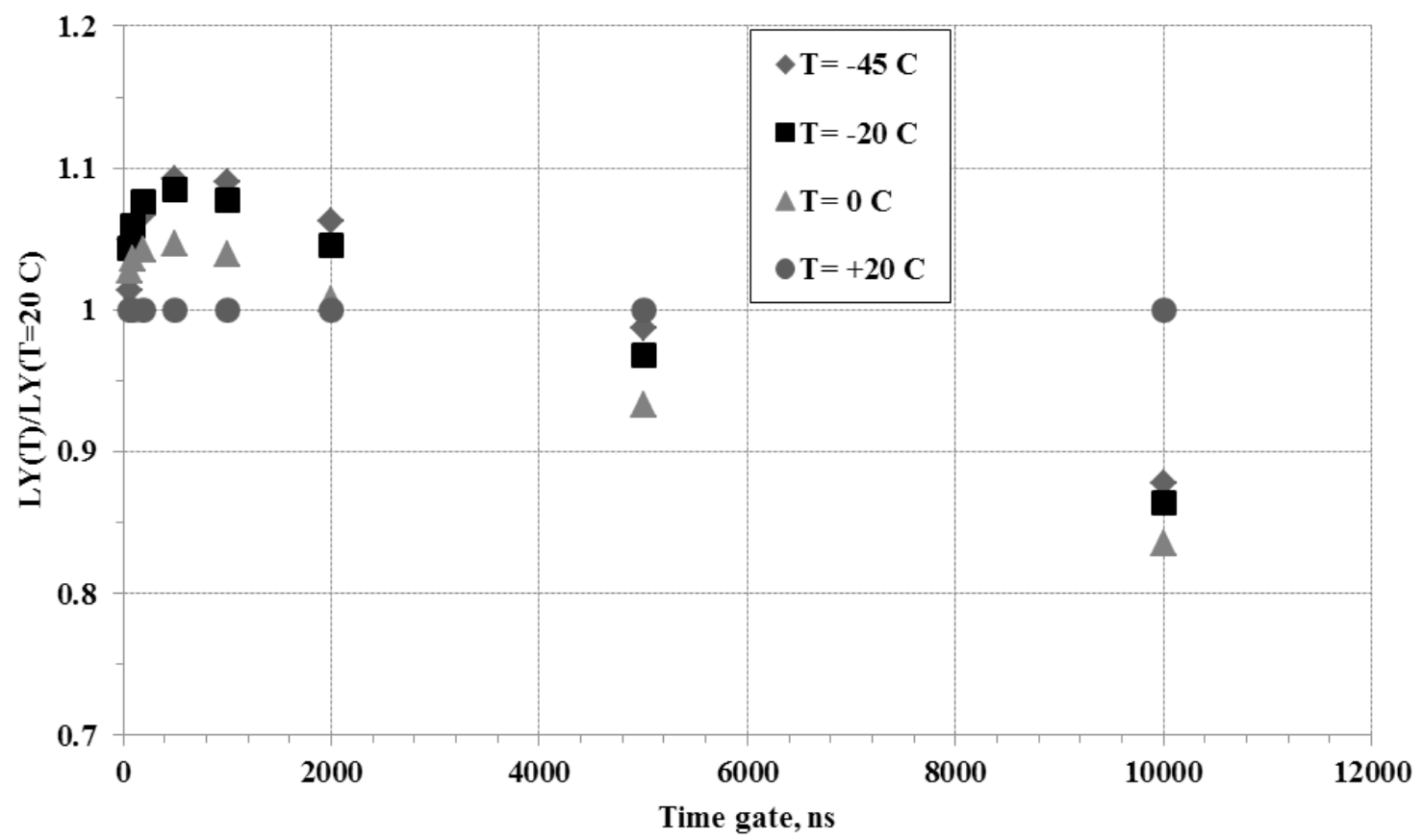

132

133

Fig.6. Relative change of the light yield of GAGG:Ce (S1) sample normalized to $20^{\circ} \mathrm{C}$

134 versus time gate at different temperatures. 


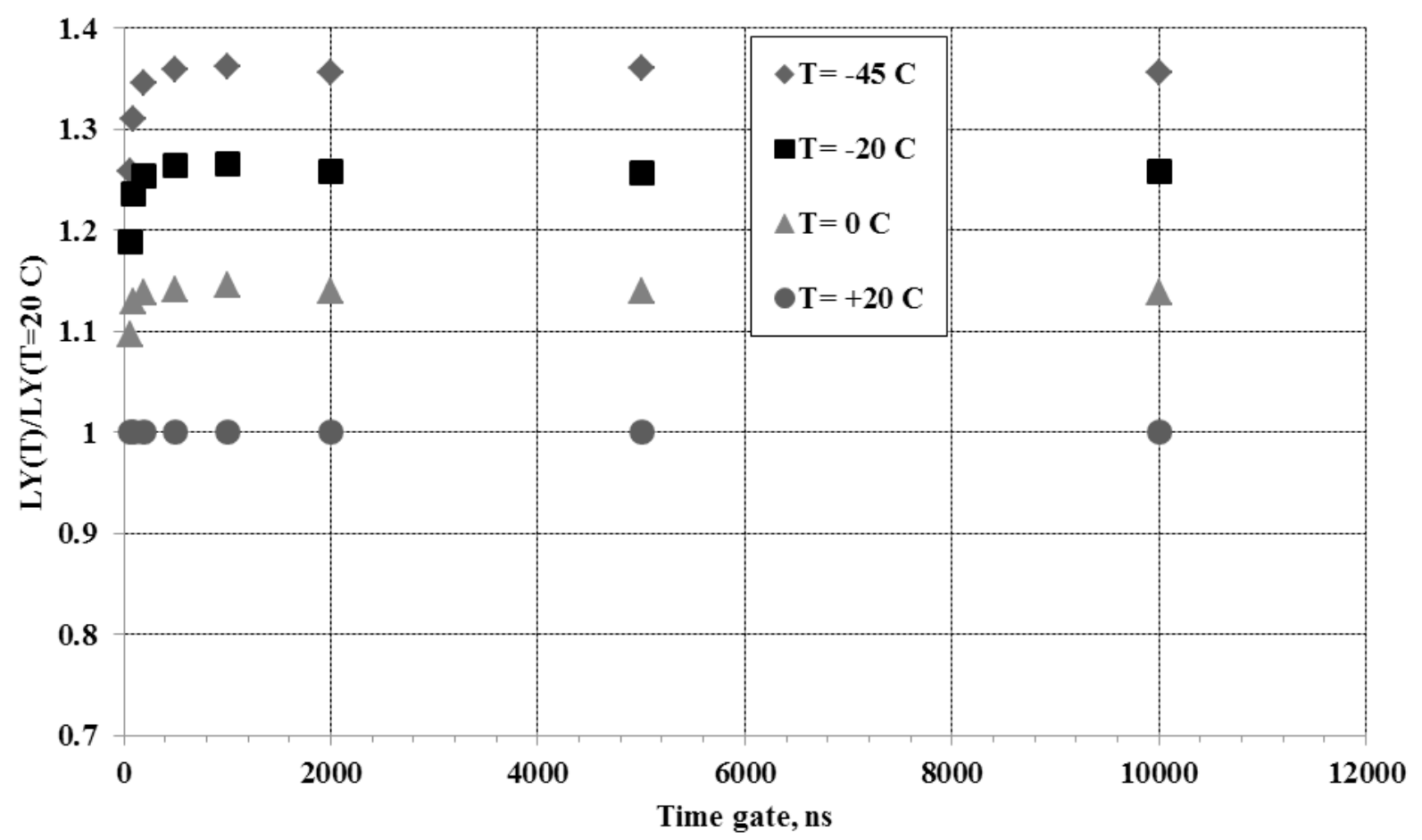

Fig.7. Relative change of the light yield of GAGG:Ce (S2) sample normalized to $20^{\circ} \mathrm{C}$ versus time gate at different temperatures.

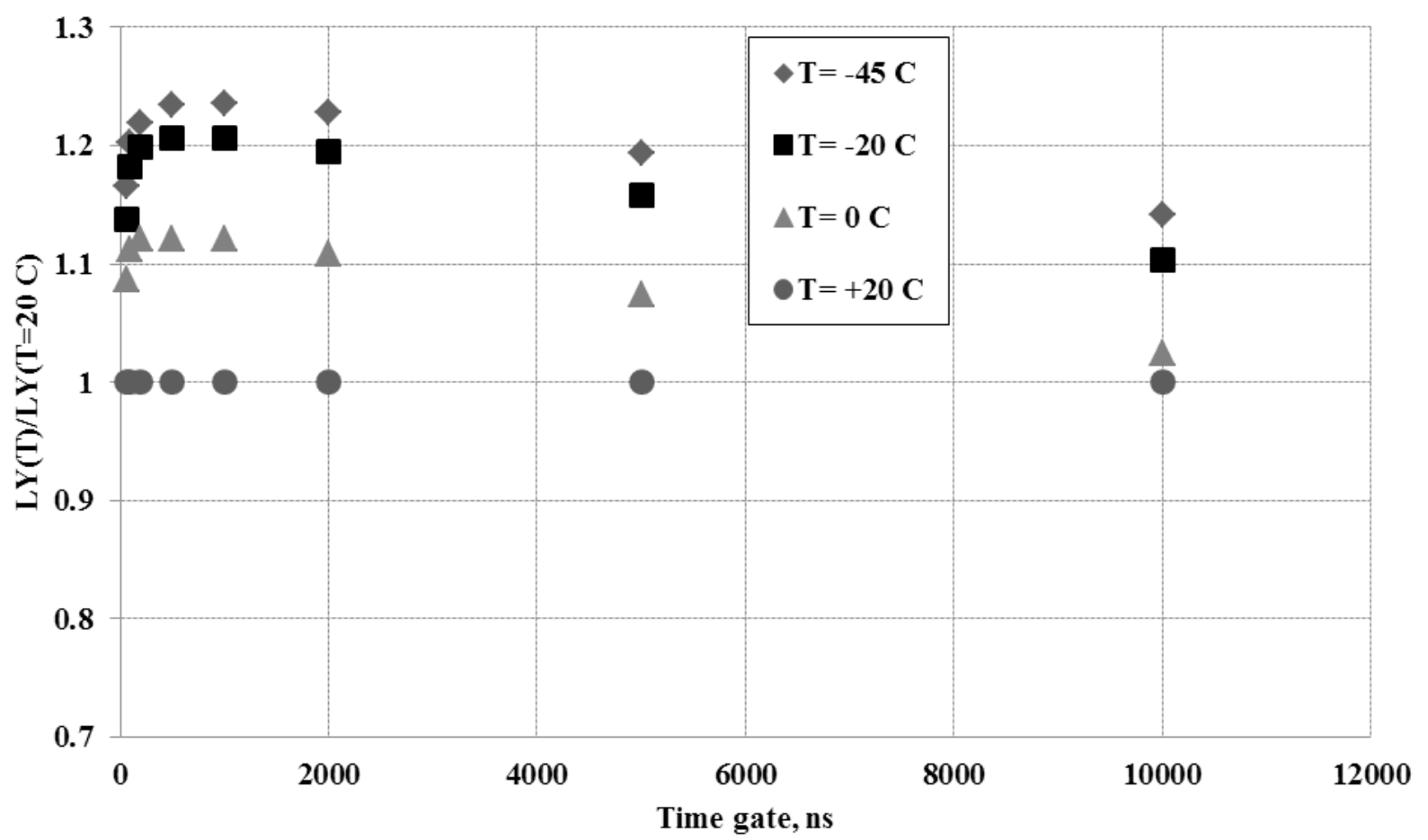

Fig.8. Relative change of the light yield of GAGG:Ce (S3) sample normalized to $20^{\circ} \mathrm{C}$ versus time gate at different temperatures. 
As it is seen, crystal doped only with Ce shows the lesser relative light yield increase at cooling. Moreover, behavior of the gated light yield shows that with temperature decrease a redistribution of the scintillation in the favour of slow components and phosphorescence occurs. Due to this reason, number of the detected photons in a long gate, for example 10 microseconds, is $20 \%$ less at $-45^{\circ} \mathrm{C}$ than at room temperature. Situation is drastically improved in co-doped crystals. More than $95 \%$ of the scintillation is collected in $300 \mathrm{~ns}$, a weak dependence of the light yield on the gate is observed. This indicates that electron traps have a minor contribution to the farther stage of the scintillation kinetics and phosphorescence as well.

\section{Conclusions}

We observed a significant improvement of the detecting properties of GAGG/PMT based scintillation detectors at their cooling. Improvement that is even more spectacular is expected at the application of SiPM for the scintillation photon detection. SiPM noise is reduced by factor two for each $10^{\circ} \mathrm{C}$ of temperature decrease, therefore combining of multidoped GAGG and SiPM readout opens an opportunity to create advantageous detectors, particularly for medical imaging applications.

\section{References}

1. K. Kamada, T. Yanagida, J. Pejchal, M. Nikl, T. Endo, K. Tsukumi, Y. Fujimoto, A. Fukabori, A. Yoshikava, Crystal Growth and Scintillation properties of Ce doped Gd3(Ga,Al)5O12 Single Crystals, IEEE Trans. Nucl. Sci. 59 (2012) 2112-2115

2. M.T. Lucchini, V. Babin, P. Bohacek, S. Gundacker, K. Kamada, M. Nik1, A. Petrosyan, A. Yoshikawa, E. Auffray, Effect of $\mathrm{Mg} 2+$ ions co-doping on timing performance and radiation tolerance of Cerium doped Gd3A12Ga3O12 crystals, Nucl. Instrum. Methods Phys. Res. A 816 (2016) 176-183

3. M.T. Lucchini, S. Gundacker, P. Lecoq, A. Benaglia, M. Nikl, K. Kamada, A. Yoshikawa, E.Auffray, Timing capabilities of garnet crystals for detection of high energy charged particles. Nucl. Instr. Meth. Phys, Research A 852,(2017)1-9

4. P.Lecoq, A.Gektin, M.Korzhik, Inorganic Scintillators for Detector Systems, Springer, 2017, P.408

5. K. Kamada, M. Nikl, S. Kurosawa, A. Beitlerova, A. Nagura; Y. Shoji; J. Pejchal, Y. 
Ohashi, Y. Yokota, A. Yoshikawa, Alkali Earth Co-doping Effects on Luminescence and Scintillation Properties of $\mathrm{Ce}$ doped $\mathrm{Gd}_{3} \mathrm{Al}_{2} \mathrm{Ga}_{3} \mathrm{O}_{12}$ scintillator. Optical Materials 41 (2015)63-66

6. Use of codoping to modify the scintillation porperties of inorganic scintillators doped with trivalent activators, US Patent 8,617,422 B2, Dec.31,2013

7. S.Blahuta, A.Bessiere, P. Dorenbos, V.Ouspensi, Evidence and conseqences of $\mathrm{Ce}^{4+}$ in LYSO:Ce, $\mathrm{Ca}$ and LYSO:Ce, Mg single crystal for medical imaging applications, IEEE Trans. Nucl. Sci. 60 (2013)3134-3141

8. M. Koschan, K. Yang, M. Zhuravleva, C.L. Melcher, A comparison of the effect of $\mathrm{Ca}^{2+}$ codoping in cerium doped GSO with that of LSO and YSO, Journal of Crystal Growth 352 (2012) 133-136

9. E Auffray, M Korjik, MT Lucchini, S Nargelas, O Sidletskiy, G Tamulaitis, Y Tratsiak, A Vaitkevičius, Free carrier absorption in self-activated $\mathrm{PbWO}_{4}$ and Ce-doped $\mathrm{Y}_{3}\left(\mathrm{Al}_{0.25}\right.$ $\left.\mathrm{Ga}_{0.75}\right)_{3} \mathrm{O}_{12}$ and $\mathrm{Gd}_{3} \mathrm{Al}_{2} \mathrm{Ga}_{3} \mathrm{O}_{12}$ garnet scintillators, Optical Materials 58(2016) 461-465

10. A. Borisevich et. al., Lead tungstate crystal with increased light yield for the PANDA electromagnetic calorimeter. Nucl. Instr. and Meth. in Phys. Res. A537(2005)101-104 